\title{
Perfil epidemiológico das neoplasias de glândulas salivares diagnosticadas em São Luís-MA
}

Primeira submissão em 01/09/09 Última submissão em 01/09/09 Aceito para publicação em 20/10/09 Publicado em 20/10/09

\author{
Epidemiologic profile of salivary gland neoplasms diagnosed in São Luis-MA
}

Rafael Sereno Loiola'; Felipe Rodrigues de Matos²; Cassiano Francisco Weege Nonaka3; Fernanda Ferreira Lopes ${ }^{4}$; Maria Carmen Fontoura Nogueira da Cruz ${ }^{5}$

\begin{abstract}
unitermos
resumo

Epidemiologia

Introdução e objetivo: As neoplasias de glândulas salivares constituem um grupo de lesões, clínica e morfologicamente diverso, capaz de determinar importantes desafios diagnósticos e terapêuticos. O escopo do

Neoplasias bucais trabalho é determinar a frequência relativa e a distribuição das neoplasias de glândulas salivares diagnosticadas Doenças das glândulas no Instituto Maranhense de Oncologia Aldenora Bello (IMOAB). Material e métodos: Realizou-se estudo retrospectivo dos casos de neoplasia de glândula salivar diagnosticados no IMOAB, no período de janeiro de 1997 a dezembro de 2007. Dados sobre sexo, idade e localização anatômica foram obtidos em prontuários médicos. Cortes histológicos foram avaliados sob microscopia de luz e os casos foram categorizados segundo a classificação da Organização Mundial da Saúde (OMS) $)^{(4)}$. Os dados coletados foram analisados por meio de estatística descritiva. Resultados: Foram identificados 232 casos, dos quais 178 eram neoplasias benignas $(76,7 \%)$ e 54 (23,3\%), malignas. Os três tipos histológicos mais frequentes foram: adenoma pleomórfico $(59,5 \%)$, tumor de Warthin (13,8\%) e carcinoma adenoide cístico (6,9\%). A maioria dos casos foi diagnosticada em pacientes do sexo feminino, com proporção homem:mulher de 1:1,3. As neoplasias benignas e malignas apresentaram picos de incidência na quarta e sétima décadas de vida, respectivamente. Com relação à localização anatômica, 154 casos $(66,4 \%)$ afetaram a parótida, 43 (18,5\%) acometeram a glândula submandibular e 35 $(15,1 \%)$ envolveram glândulas salivares menores. Conclusão: Em conjunto com outros estudos, os resultados da presente pesquisa sugerem discretas variações na frequência relativa e distribuição das neoplasias de glândulas salivares entre as populações do Brasil e de outras regiões do mundo.
\end{abstract}

abstract

Introduction and objective: The salivary gland neoplasms are a clinically and morphologically diverse group of lesions able to determine important diagnostic and therapeutic challenges. The objective of this study is to determine the relative frequency and distribution of salivary gland neoplasms diagnosed at Aldenora Bello Institute of Oncology - Maranhão State (Instituto Maranhense de Oncologia Aldenora Bello [IMOAB]). Material and methods: It was performed a retrospective analysis of the salivary gland neoplasms diagnosed at IMOAB between January 1997 and December 2007. Data regarding gender, age, and anatomic site were retrieved from medical records. Histological slides were evaluated by light microscopy and cases were categorized according to the World Health Organization classification ${ }^{(4)}$. The collected data were analyzed through descriptive statistics. Results: A total of 232 cases were identified, of which 178 were benign neoplasms (76.7\%) and 54 (23.3\%) were malignant. The three most frequent histological types were pleomorphic adenoma (59.5\%), Warthin's tumor (13.8\%) and adenoid cystic carcinoma (6.9\%). Most cases were diagnosed in females, with a male-female ratio of 1:1.3. The benign and malignant neoplasms showed incidence peaks at the 4th and 7th decades of life, respectively. Regarding anatomic site, 154 cases (66.4\%) affected the parotid gland, 43 (18.5\%) affected the submandibular gland and 35 (15.1\%) involved minor salivary glands. Conclusion: In association with other studies, the present results suggest slight variations in the relative frequency and distribution of salivary gland neoplasms between Brazilian and worldwide populations. key words

Epidemiology

Mouth neoplasms

Salivary glands diseases

1. Cirurgião-dentista pela Universidade Federal do Maranhão (UFMA).

2. Cirurgião-dentista; mestrando do programa de pós-graduação em Patologia Oral da Universidade Federal do Rio Crande no Norte (UFRN).

3. Mestre em Patologia Oral; doutorando do programa de pós-graduação em Patologia Oral da UFRN.

4. Doutora em Patologia Oral pela UFRN; professora da disciplina de Semiologia do Departamento de Odontologia II da UFMA.

5. Doutora em Patologia Oral pela UFRN; professora da disciplina de Patologia Oral do Departamento de Odontologia II da UFMA. 


\section{Introdução}

As neoplasias de glândulas salivares constituem um grupo de lesões, clínica e morfologicamente diverso, capaz de determinar importantes desafios diagnósticos e terapêuticos $^{(1,6,7,21)}$. A incidência estimada para essas lesões é de 0,4 a 3,5 casos por grupo de 100 mil pessoas, constituindo menos de $5 \%$ das neoplasias da região de cabeça e pescoço em adultos e cerca de $8 \%$ em crianças $^{(1,2,7,8)}$.

A maioria dos casos é diagnosticada em mulheres $^{(1,2,10,12,17,19,20)}$, com percentuais que variam entre $54 \%(1)$ e $62 \%{ }^{(14)}$. Considerando todos os tumores de glândulas salivares, a média de idade dos pacientes ao diagnóstico é de 45 anos ${ }^{(22)}$. Quando analisadas isoladamente, neoplasias benignas e malignas exibem variações na distribuição segundo a faixa etária. A maioria dos tumores benignos é observada em pacientes na terceira década de vida, e a maior proporção dos tumores malignos é identificada em indivíduos na sexta década ${ }^{(1)}$.

Variações geográficas têm sido observadas nesse grupo de lesões, particularmente em relação a localização anatômica e subtipos histológicos $(3,13,15)$. Embora a maioria dos estudos identifique a glândula parótida como sítio mais comum para essas lesões ${ }^{(1-3,10,13,14,16-20,22)}$, pesquisas divergem sobre o segundo sítio mais frequente. Estudos realizados no Irã (2), na Nigéria( ${ }^{(3)}$, em Ibadan ${ }^{(13)}$ e no Brasi $^{(17,19)}$ identificam a glândula submandibular como o segundo sítio mais acometido, ao passo que pesquisas conduzidas na Jordânia(1) e na Índia(18) sugerem que as glândulas salivares menores o são. Por sua vez, um estudo demográfico realizado no Reino Unido(12) identificou as glândulas salivares menores como o sítio mais frequente para essas lesões.

Os tumores benignos são mais comuns, constituindo entre $54 \%$ e $79 \%$ dos casos, enquanto os malignos representam $21 \%$ a $46 \%$ das neoplasias $(10,12,19,20,22)$. $O$ adenoma pleomórfico tem sido descrito como o tumor de glândula salivar mais comum, perfazendo $33,2 \%$ a $89,9 \%$ dos $\operatorname{casos}^{(1,11,12,16,18,20,22)}$, seguido dos carcinomas adenoide cístico e mucoepidermoide ${ }^{(9,15,18,20)}$. No entanto, trabalhos realizados no Brasil identificaram o tumor de Warthin como a segunda ${ }^{(14,17,19)}$ ou terceira ${ }^{(10)}$ neoplasia de glândula salivar mais comum. Pesquisas desenvolvidas no Reino Unido ${ }^{(12)}$, na Nigéria ${ }^{(13)}$ e na Índia ${ }^{(18)}$ reportaram baixas frequências para o tumor de Warthin, com percentuais entre $2,5 \%$ e $4,6 \%$, constituindo apenas a sexta ${ }^{(12)}$ ou sétima ${ }^{(16,18)}$ neoplasia de glândula salivar mais frequente.
O presente estudo tem como objetivo determinar a frequência relativa e a distribuição das neoplasias de glândulas salivares diagnosticadas no Instituto Maranhense de Oncologia Aldenora Bello (IMOAB), no período de janeiro de 1997 a dezembro de 2007, comparando os resultados obtidos com os reportados na literatura.

\section{Material e métodos}

Foi realizado um estudo retrospectivo dos casos de neoplasias de glândulas salivares, diagnosticados no IMOAB durante o período de janeiro de 1997 a dezembro de 2007. O projeto de pesquisa que originou este trabalho foi submetido à apreciação do Comitê de Ética em Pesquisa da Universidade Federal do Maranhão (UFMA), sendo aprovado conforme parecer no 2008-37.

Foram coletados os dados referentes a sexo e idade dos pacientes e localização anatômica das lesões. Para o estudo histopatológico foram utilizadas lâminas contendo cortes com $5 \mu \mathrm{m}$ de espessura corados pela técnica de rotina da hematoxilina e eosina (HE), mantidas em arquivo. Todos os casos foram avaliados sob microscopia de luz, sendo classificados de acordo com os critérios propostos pela Organização Mundial da Saúde (OMS) ${ }^{(4)}$.

Os dados obtidos foram tabulados em planilhas eletrônicas utilizando-se o software Microsoft Excel (Microsoft Corporation) e posteriormente exportados para o software Statistical Package for the Social Sciences (SPSS 13.0), no qual foram obtidos os percentuais, médias e frequências absolutas por meio de técnicas de estatística descritiva.

\section{Resultados}

Foram identificadas 232 neoplasias de glândulas salivares, que corresponderam a $0,25 \%$ de todos os casos registrados no IMOAB no período de janeiro de 1997 a dezembro de 2007. As neoplasias benignas perfizeram a maior casuística no levantamento, com 178 casos $(76,7 \%)$, enquanto as malignas foram representadas por 54 casos (23,3\%). O adenoma pleomórfico foi o subtipo histológico mais frequente, com 138 registros $(59,5 \%)$, seguido pelo tumor de Warthin, com 32 casos $(13,8 \%)$ e pelo carcinoma adenoide cístico, com 16 casos (6,9\%). A distribuição 
das neoplasias de acordo com o subtipo histológico está apresentada na Tabela 1.

\begin{tabular}{|c|c|c|}
\hline \multirow{2}{*}{$\begin{array}{c}\text { ravela } 1 \\
\text { Tino histológico }\end{array}$} & \multirow[b]{2}{*}{ Número (n) } & \multirow{2}{*}{$\begin{array}{l}\text { sias benignas } \\
\text { o histológico } \\
\text { Percentual (\%) }\end{array}$} \\
\hline & & \\
\hline Adenoma pleomórfico & 138 & 59,5 \\
\hline Tumor de Warthin & 32 & 13,8 \\
\hline $\begin{array}{l}\text { Adenoma de células } \\
\text { basais }\end{array}$ & 3 & 1,3 \\
\hline Oncocitoma & 3 & 1,3 \\
\hline Adenoma canalicular & 2 & 0,9 \\
\hline $\begin{array}{l}\text { Carcinoma adenoide } \\
\text { cístico }\end{array}$ & 16 & 6,9 \\
\hline $\begin{array}{l}\text { Carcinoma } \\
\text { mucoepidermoide }\end{array}$ & 14 & 6 \\
\hline $\begin{array}{l}\text { Carcinoma ex- } \\
\text { adenoma pleomórfico }\end{array}$ & 8 & 3,4 \\
\hline Adenocarcinoma NOS & 8 & 3,4 \\
\hline $\begin{array}{l}\text { Carcinoma de células } \\
\text { acinares }\end{array}$ & 4 & 1,8 \\
\hline $\begin{array}{l}\text { Carcinoma de células } \\
\text { escamosas }\end{array}$ & 2 & 0,9 \\
\hline $\begin{array}{l}\text { Carcinoma de células } \\
\text { claras NOS }\end{array}$ & 1 & 0,4 \\
\hline $\begin{array}{l}\text { Carcinoma } \\
\text { linfoepitelial }\end{array}$ & 1 & 0,4 \\
\hline Total & 232 & 100 \\
\hline
\end{tabular}

NOS: não especificado de outra forma.

Em relação ao sexo, dos 232 casos diagnosticados, 134 $(57,8 \%)$ foram observados em indivíduos do sexo feminino e 98 (42,2\%) do masculino, determinando uma proporção entre homens e mulheres de 1:1,3. Considerando apenas as neoplasias benignas, houve 106 casos $(59,6 \%)$ em mulheres e 72 (40,4\%) em homens (Tabela 2). Analisando apenas as neoplasias malignas, 28 casos (51,9\%) foram diagnosticados em pacientes do sexo feminino e $26(48,1 \%)$ do sexo masculino (Tabela 3). A maioria das neoplasias benignas foi diagnosticada entre a quarta e sexta décadas de vida (Tabela 4), ao passo que as malignas revelaram pico de incidência na sétima década (Tabela 5).

Quanto à localização anatômica, a glândula parótida foi o sítio acometido com maior frequência, com 154 casos $(66,4 \%)$ registrados, seguido da glândula submandibular,
Distribuição das neoplasias benignas das glândulas salivares quanto ao tipo

Tabela 2 histológico e ao sexo (São Luís-MA, 2008)

Sexo

Tipo

histológico

Feminino $n$ Masculino $n$ Total $n(\%)$ $(\%)$

Adenoma

pleomórfico

$85(80,2)$

$53(73,6)$

$138(77,5)$

Tumor de

Warthin

$16(15,1) \quad 16(22,2)$

32 (18)

Adenoma

de células

$2(1,9)$

$1(1,4)$

$3(1,7)$

basais

Oncocitoma $2(1,9) \quad 1(1,4) \quad 3(1,7)$

Adenoma

canalicular

$1(0,9)$

$1(1,4)$

$2(1,1)$

Total

$106(100) \quad 72(100)$

$178(100)$
Tabela 3

\section{Tipo histológico}

Carcinoma

adenoide cístico

Carcinoma

mucoepidermoide

Carcinoma

ex-adenoma

pleomórfico

Adenocarcinoma

NOS

Carcinoma de

células acinares

Carcinoma

de células

escamosas

Carcinoma de

células claras NOS

Carcinoma

linfoepitelial

Total

28 (100)

NOS: não especificado de outra forma.

$6(21,5)$

$2(7,1)$
Distribuição das neoplasias malignas das glândulas salivares quanto ao tipo histológico e ao sexo (São Luís-MA, 2008)

\begin{tabular}{ccc}
\multicolumn{2}{c}{ Sexo } & Total \\
\cline { 1 - 2 } Feminino & Masculino & $n(\%)$ \\
$n(\%)$ & $n(\%)$ &
\end{tabular}

14 $(25,9)$

$8(28,6) \quad 6(23,1)$

$2(7,7)$

$8(14,8)$

$6(23,1)$

$8(14,8)$

$2(7,1) \quad 2(7,7) \quad 4(7,4)$

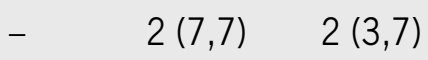

$1(3,6) \quad--\quad 1(1,9)$

$1(3,8) \quad 1(1,9)$

26 (100) 54 (100) 
Distribuição das neoplasias benignas das glândulas salivares quanto ao tipo histológico e à idade

Tabela 4 dos pacientes (São Luís-MA, 2008)

\begin{tabular}{|c|c|c|c|c|c|c|c|c|c|c|c|}
\hline \multirow{2}{*}{$\begin{array}{c}\text { Tipo } \\
\text { histológico }\end{array}$} & \multicolumn{11}{|c|}{ Idade em décadas } \\
\hline & $1-10$ & $11-20$ & $21-30$ & $31-40$ & $41-50$ & $51-60$ & $61-70$ & $71-80$ & $81-90$ & Total & $n(\%)$ \\
\hline $\begin{array}{l}\text { Adenoma } \\
\text { pleomórfico }\end{array}$ & 2 & 12 & 26 & 31 & 26 & 22 & 11 & 4 & 3 & $\begin{array}{c}137 \\
(78,7)\end{array}$ & \\
\hline $\begin{array}{l}\text { Tumor de } \\
\text { Warthin }\end{array}$ & - & - & - & 8 & 5 & 8 & 7 & 2 & - & $\begin{array}{c}30 \\
(17,3)\end{array}$ & \\
\hline $\begin{array}{l}\text { Adenoma } \\
\text { de células } \\
\text { basais }\end{array}$ & - & - & - & - & 2 & - & - & 1 & - & $3(1,7)$ & \\
\hline Oncocitoma & - & - & - & 1 & 1 & 1 & - & - & - & $3(1,7)$ & \\
\hline $\begin{array}{l}\text { Adenoma } \\
\text { canalicular }\end{array}$ & - & - & - & - & - & 1 & - & - & - & $1(0,6)$ & \\
\hline Total & 2 & 12 & 26 & 40 & 34 & 32 & 18 & 7 & 3 & $\begin{array}{l}174^{*} \\
(100)\end{array}$ & \\
\hline
\end{tabular}

*A informação não estava disponível em um caso de adenoma pleomórfico, um caso de adenoma canalicular e dois casos de tumor de Warthin.

Tabela 5

\section{Tipo histológico}

Carcinoma

adenoide cístico

Carcinoma

mucoepidermoide

Carcinoma

ex-adenoma

pleomórfico

Adenocarcinoma

NOS

Carcinoma de células acinares

Carcinoma de células escamosas

Carcinoma de células claras NOS

Carcinoma

linfoepitelial

Total
Distribuição das neoplasias malignas das glândulas salivares quanto ao tipo histológico e à idade dos pacientes (São Luís-MA, 2008)

Idade em décadas

* Informação não estava disponível em dois casos de carcinoma de células acinares. NOS: não especificado de outra forma. 
com 43 casos (18,5\%) e das glândulas menores, com 35 casos (15,1\%). A análise isolada das neoplasias benignas revelou a glândula parótida como sítio mais comum, com 129 registros (72,5\%), seguida da glândula submandibular, com 36 casos $(20,2 \%)$, e pelas glândulas menores, com 13 registros (7,3\%) (Tabela 6). Com relação às neoplasias malignas, a glândula parótida também se apresentou como sítio mais acometido, perfazendo 25 casos (46,3\%), seguida pelas glândulas salivares menores, com 22 casos $(40,7 \%)$, e pela submandibular, com sete casos (13\%) (Tabela 7).
Tabela 6

\section{Tipo histológico}

Adenoma

pleomórfico

Tumor de Warthin

Adenoma de

células basais

Oncocitoma

Adenoma

canalicular

Total

\section{localização anatômica (São Luís-MA, 2008) \\ Glândula salivar}

Distribuição das neoplasias benignas das glândulas salivares quanto ao tipo histológico e a

Parótida $n(\%) \quad$ Submandibular $n(\%) \quad$ Sublingual $n(\%) \quad$ Menores $n(\%) \quad$ Total $n(\%)$

$91(70,5) \quad 34(94,4)$

13 (100)

$138(77,5)$

$2(5,6)$

$32(18)$

$3(2,3)$

$3(2,3)$

$2(1,6)$

$3(1,7)$

$3(1,7)$

$2(1,1)$

$129(100)$

36 (100)

$13(100)$

$178(100)$

Distribuição das neoplasias malignas das glândulas salivares quanto ao tipo histológico e à

Tabela 7

localização anatômica (São Luís-MA, 2008)

\section{Tipo histológico}

Carcinoma

adenoide cístico

Carcinoma

mucoepidermóide

Carcinoma

ex-adenoma

pleomórfico

Adenocarcinoma

NOS

Carcinoma de

células acinares

Carcinoma

de células

escamosas

Carcinoma de

células claras

NOS

Carcinoma

linfoepitelial

Total

\section{Glândula salivar}

Parótida $n(\%) \quad$ Submandibular $n(\%) \quad$ Sublingual $n(\%) \quad$ Menores $n(\%) \quad$ Total $n(\%)$

$\begin{array}{lllll}4(16) & 3(42,8) & - & 9(40,9) & 16(29,6)\end{array}$

5 (20)

$1(14,3)$

$8(36,4)$

$14(25,9)$

$7(28)$

$1(4,5)$

$8(14,8)$

3 (12)

$2(28,6)$

$3(13,7)$

$8(14,8)$

2 (8)

$1(14,3)$

$1(4,5)$

$4(7,4)$

2 (8)

1 (4)

$1(1,9)$

1 (4)

$1(1,9)$

25 (100)

7 (100)

$22(100)$

54 (100)

NOS: não especificado de outra forma. 


\section{Discussão}

A despeito dos diversos estudos retrospectivos já publicados, a frequência relativa e a distribuição das neoplasias de glândulas salivares permanecem temas de discussão na literatura científica. No presente levantamento, a amostra representou $0,25 \%$ do total de lesões diagnosticadas no IMOAB. Estudos realizados em outros serviços revelam que as neoplasias de glândulas salivares podem constituir de $0,15 \%^{(14)}$ a $10 \%{ }^{(3)}$ do total de lesões diagnosticadas.

$\mathrm{Na}$ atual pesquisa, as neoplasias benignas foram mais comuns, respondendo por $76,7 \%$ de todos os casos de neoplasia de glândula salivar. Outros estudos realizados no Brasil também observaram maior frequência de neoplasias benignas, com percentuais entre $76,3 \%$ e $79,8 \%(14,17,19)$. No entanto, pesquisas realizadas na África verificaram percentuais comparativamente menores para as neoplasias benignas, variando entre $54,1 \%$ e $55,7 \%{ }^{(16,20)}$. No presente estudo, os tumores malignos constituíram $23,3 \%$ da amostra, corroborando os resultados apresentados por outras pesquisas conduzidas no Brasi ${ }^{(14,17,19)}$. Contudo, estudos realizados na Índia e na África constataram percentuais comparativamente maiores para os tumores malignos, variando entre $38 \%$ e $45,9 \%(16,18,20)$.

No presente levantamento, os tumores de glândulas salivares apresentaram maior acometimento do sexo feminino $(57,8 \%)$, com uma proporção entre homens e muIheres de 1:1,3. Este resultado corrobora a predileção pelo sexo feminino descrita em outros estudos $(1,2,12,14,17,19,20)$, nos quais $54 \%$ a $62,4 \%$ dos indivíduos acometidos eram mulheres. Todavia, Subhashraj ${ }^{(18)}$ evidenciou percentual discretamente maior em pacientes do sexo masculino (51\%). Na presente pesquisa, a análise isolada das neoplasias benignas revelou maior acometimento do sexo feminino (59,6\%). De forma similar, estudos em séries de casos relataram maior proporção de tumores benignos em mulheres $(54,46 \% \text { a } 65,16 \%)^{(2,10,12,14,19,20)}$.

No estudo ora realizado, a avaliação exclusiva das neoplasias malignas revelou acometimento discretamente maior de pacientes do sexo feminino (51,9\%). Análises em outras populações do Brasil(14, 19) e de outros países $^{(1,12,20)}$ também relatam maiores percentuais de neoplasias malignas em mulheres, variando entre $52,45 \%$ e $66,67 \%$. Porém, alguns trabalhos reportaram discreta predileção dos tumores malignos de glândulas salivares por indivíduos do sexo masculino ${ }^{(2,10,18)}$, variando entre $51,22 \%$ e $52,2 \%$.
Com relação à idade, a maior proporção das neoplasias benignas $(61,5 \%)$ foi diagnosticada em indivíduos entre a quarta e a sexta década de vida. Coerentemente, Ito et al. ${ }^{(10)}$ e Subhashraj ${ }^{(18)}$ verificaram picos de incidência para esse grupo de tumores entre a quarta e a quinta década de vida e entre a quarta e a sexta décadas, respectivamente. Por sua vez, Al-Khateeb e Ababneh ${ }^{(1)}$ e Otoh et al. ${ }^{(16)}$ observaram picos de incidência para as neoplasias benignas entre a segunda e quarta décadas de vida e entre a terceira e a quarta décadas, respectivamente. No presente estudo, as neoplasias malignas exibiram pico de incidência na sétima década de vida. Resultados similares foram reportados por Ito et al. ${ }^{(10)}$ e Lima et al.(14). Contudo, Otoh et al. ${ }^{(16)}$ observaram maior proporção das neoplasias malignas entre a quarta e a sexta década de vida. Adicionalmente, Al-Khateeb e Ababneh ${ }^{(1)}$ identificaram dois picos distintos para os tumores malignos de glândulas salivares, situados na quarta e sétima décadas de vida.

Em relação à localização anatômica, no presente estudo o sítio acometido com maior frequência foi a glândula parótida $(66,4 \%)$. Coerentemente, a maioria dos estudos retrospectivos descreve essa glândula como a localização anatômica mais comum para os tumores de glândulas salivares, estando afetada em $34 \%$ a $80 \%$ dos $\operatorname{casos}^{(1,2,10,14,16-20,22)}$. O segundo sítio anatômico mais frequente neste levantamento foi a glândula submandibular (18,5\%). Pesquisas realizadas no Irã ${ }^{(2)}$ e em algumas regiões do Brasil(17, 19) também verificaram a glândula submandibular como o segundo sítio mais acometido, com percentuais entre $19,3 \%$ e $24,19 \%$. Entretanto, estudos conduzidos na Índia ${ }^{(18)}$, na Jordânia ${ }^{(1)} \mathrm{e}$ na Nigéria ${ }^{(16)}$ identificaram as glândulas salivares menores como a segunda localização mais comum, estando afetadas em $22 \%, 28 \%$ e $41,02 \%$ dos casos, respectivamente. Já um estudo realizado em Uganda ${ }^{(20)}$ reportou distribuição relativamente similar dos tumores entre as glândulas parótida (34\%), submandibular (33\%) e salivares menores (32\%). Diferentemente, um estudo demográfico realizado no Reino Unido ${ }^{(12)}$ identificou as glândulas salivares menores como sítio mais comum para as neoplasias de glândula salivar. Se as diferenças nos resultados desses estudos decorrem de características peculiares das populações analisadas ou de particularidades dos serviços nos quais foram conduzidas as pesquisas, ainda é assunto de discussão.

Considerando todos os tumores de glândulas salivares, os três tipos histológicos descritos com maior frequência são o adenoma pleomórfico, o carcinoma mucoepidermoide e o carcinoma adenoide cístico ${ }^{(1,11,12,15,16,18,20,22)}$. $\mathrm{O}$ adenoma pleomórfico é o tumor de glândula salivar 
mais comum, constituindo entre $33,2 \%$ e $89,9 \%$ das neoplasias $(1,11,12,16,18,20,22)$. Os achados do presente estudo corroboram essa afirmação. Na presente pesquisa, o tumor de Warthin foi a segunda neoplasia de glândula salivar mais frequente $(13,8 \%)$, com a maioria dos casos localizados em glândula parótida $(93,8 \%)$. Coerentemente, outros estudos retrospectivos realizados no Brasil descreveram o tumor de Warthin como a segunda ${ }^{(14,17,19)}$ ou terceira ${ }^{(10)}$ neoplasia de glândula salivar mais comum. Porém trabalhos desenvolvidos no Reino Unido(12), na Nigéria ${ }^{(16)}$ e na Índia ${ }^{(18)}$ reportaram baixas frequências para o tumor de Warthin, com percentuais entre $2,5 \%$ e $4,6 \%$, constituindo apenas a sexta ${ }^{(12)}$ ou sétima ${ }^{(16,18)}$ neoplasia de glândula salivar mais frequente. Em conjunto com outros estudos realizados no Brasil, os resultados da presente pesquisa sugerem diferenças na frequência do tumor de Warthin entre essa e outras populações. Novos estudos são necessários para avaliar tal hipótese.

O carcinoma adenoide cístico foi a terceira neoplasia de glândula salivar mais frequente $(6,9 \%)$, corroborando os achados relatados por Ito et al. ${ }^{(10)}$, Jones et al. ${ }^{(12)}$, Santos et al..$^{(17)}$ e Vargas et al. ${ }^{(19)}$. Entretanto Subhashraj ${ }^{(18)}$ e Vuhahula ${ }^{(20)}$ descreveram essa lesão como a segunda neoplasia de glândula salivar mais comum, constituindo $9,64 \%$ e $13,43 \%$ dos casos, respectivamente. A despeito da possibilidade de influências éticas ou geográficas, é provável que essa variação na frequência do carcinoma adenoide cístico seja decorrente de diferenças nos critérios diagnósticos para essa neoplasia ${ }^{(5)}$. De acordo com Buchner et al. ${ }^{(5)}$, a alta prevalência do carcinoma adenoide cístico pode estar associada à falta de familiaridade de alguns patologistas com o diagnóstico do adenocarcinoma polimorfo de baixo grau, uma neoplasia que compartilha características histopatológicas com o carcinoma adenoide cístico.

De maneira geral, os resultados do presente trabalho corroboram os relatados na literatura. Em conjunto com outros estudos, os dados da presente pesquisa sugerem discretas variações na frequência relativa e distribuição das neoplasias de glândulas salivares entre as populações do Brasil e de outras regiões do mundo. Novas pesquisas são necessárias para esclarecer se tais diferenças decorrem de características peculiares das populações analisadas ou de particularidades dos serviços nos quais foram conduzidos os estudos.

\section{Referências}

1. AL-KHATEEB, T. H.; ABABNEH, K. T. Salivary tumors in north Jordanians: a descriptive study. Oral Surg Oral Med Oral Pathol Oral Radiol Endod, v.103, p. 53-9, 2007.

2. ANSARI, M. H. Salivary gland tumors in an Iranian population: a retrospective study of 130 cases. J Oral Maxillofac Surg, v. 65, p. 2187-94, 2007.

3. AROTIBA, G. T. Salivary gland neoplasms in Lagos, Nigeria. West Afr J Med, v. 15, n. 1, p. 11-7, 1996.

4. BARNES, L. et al. World Health Organization Classification of Tumours. Pathology and Genetics of Head and Neck Tumours. Lyon: IARCPress, 2005.

5. BUCHNER, A.; MERRELL, P. W.; CARPENTER, W. $M$. Relative frequency of intra-oral minor salivary gland tumors: a study of 380 cases from northern California and comparison to reports from other parts of the world. J Oral Pathol Med, v. 36, p. 207-14, 2007.

6. CARDOSO, S. V. et al. Warthin's tumor at the Brazilian National Cancer Institute: additional evidence of homogeneous sex prevalence and association with other neoplasms. ORL, v. 70, p. 339-43, 2008.
7. CHEN, A. M. et al. Late recurrence from salivary gland cancer: when does "cure" mean cure? Cancer, v. 112, p. 340-4, 2008.

8. ELLIES, M.; ARGLEBE, C.; LASKAWI, R. Tumors of the salivary glands in childhood and adolescence. J Oral Maxillofac Surg, v. 64, p. 1049-58, 2006.

9. GOODE, R. K.; AUCLAIR, P. L.; ELLIS, G. L. Mucoepidermoid carcinoma of the major salivary glands. Cancer, v. 82, n. 7, p. 1217-84, 1998.

10. ITO, F. A. et al. Salivary gland tumors in a Brazilian population: a retrospective study of 496 cases. Int J Oral Maxillofac Surg, v. 34, p. 533-6, 2005.

11. JABER, M. A. Intraoral minor salivary gland tumors: a review of 75 cases in a Libyan population. Int J Oral Maxillofac Surg, v. 35, p. 150-4, 2006.

12. JONES, A. V. et al. The range and demographics of salivary gland tumours diagnosed in a UK population. Oral Oncol, v. 44, p. 407-17, 2008.

13. KOLUDE, B.; LAWOYIN, J. O.; AKANG, E. E. Salivary gland neoplasms: a 21 year review of cases seen at University College Hospital, Ibadan. Afr J Med Med Sci, v. 30, p. 95-8, 2001. 
14. LIMA, S. S. et al. Epidemiologic profile of salivary gland neoplasms analysis of 245 cases. Rev Bras Otorrinolaringol, v. 71, n. 3, p. 335-40, 2005.

15. MASANJA, M. I.; KALYANYAMA B. M.; SIMON, E. N. Salivary gland tumours in Tanzania. East Afr Med J, v. 80, n. 8, p. 429-34, 2003.

16. OTOH, E. C. et al. Salivary gland neoplasms in Maiduguri, north-eastern Nigeria. Oral Dis, v. 11, p. 386-91, 2005.

17. SANTOS, G. C. etal. Neoplasia de glândulas salivares: estudo de 119 casos. J Bras Patol Med Lab, v. 39, n. 4, p. 371-5, 2003.

18. SUBHASHRAJ, K. Salivary gland tumors: a single institution experience in India. Br J Oral Maxillofac Surg, v. 46, p. 635-8, 2008.
19. VARGAS, P. A. et al. Salivary gland tumors in a Brazilian population: a retrospective study of 124 cases. Rev Hosp Clin Fac Med S Paulo, v. 57, n. 6, p. 271-6, 2002.

20. VUHAHULA, E. A. M. Salivary gland tumors in Uganda: clinical pathological study. Afr Health Sci, v. 4, n. 1, p. 15-23, 2004.

21. WAHLBERG, P. et al. Carcinoma of the parotid and submandibular glands: a study of survival in 2465 patients. Oral Oncol, v. 38, p. 706-13, 2002.

22. ZAMARRO, M. T. L.; ROYO, A. G.; ROYO, F. G. Afección tumoral de las glándulas salivales. Nuestra experiencia. Acta Otorrinolaringol Esp, v. 60, p. 120-5, 2009. 